\title{
Are Acute Phase Reactants and CA125 Useful for Pregnancy Prediction in Frozen Embryo Transfer?
}

\author{
Ayse Z. Ozdemir ${ }^{1}$, Cagri Gulumser ${ }^{2}$, Aysin Turkmenn', Davut Guven ${ }^{1}$, Pervin Karli3 \\ ${ }^{1}$ Ondokuz Mayıs University Hospital, Ondokuz Mayıs University IVF Center, Samsun, Turkey \\ ${ }^{2}$ Department of Obstetrics and Gynecology, University of Health Science, Istanbul, Turkey \\ ${ }^{3}$ Department of Obstetrics and Gynecology, Amasya University, Amasya, Turkey \\ Email: *aysezehra.ozdemir@hotmail.com
}

How to cite this paper: Ozdemir, A.Z., Gulumser, C., Turkmen, A., Guven, D. and Karli, P. (2019) Are Acute Phase Reactants and CA125 Useful for Pregnancy Prediction in Frozen Embryo Transfer? Advances in Reproductive Sciences, 7, 31-38. https://doi.org/10.4236/arsci.2019.72005

Received: March 10, 2019

Accepted: May 7, 2019

Published: May 10, 2019

Copyright $\odot 2019$ by author(s) and Scientific Research Publishing Inc. This work is licensed under the Creative Commons Attribution International License (CC BY 4.0).

http://creativecommons.org/licenses/by/4.0/

\begin{abstract}
Objective: This study assessed blood C-reactive protein (CRP), fibrinogen, procalcitonin, and CA125 levels and determined whether they have an effect on pregnancy. Material \& Methods: Between Feb. and Sep. 2018, 50 consecutive women who had their first frozen embryo transfer at a tertiary referral IVF center in Turkey were included in the study. Serum samples were collected on the second day of the cycle and on the transfer day. The women were divided into two groups based on the results of pregnancy outcome: pregnant (group 1) and non-pregnant (group 2). Blood CRP, fibrinogen, procalcitonin, and CA125 levels were compared between the groups. Results: There were no statistically significant differences between the groups according to CA125, CRP, fibrinogen, and procalcitonin levels at the beginning of the cycle and on the transfer day. In comparison with levels at the beginning of the cycle and on the transfer day, fibrinogen and CRP were significantly higher on the transfer day than at the beginning of the cycle. Procalcitonin was significantly higher on the transfer day than at the beginning of the cycle in group 1. However, there was no statistically significant difference between the transfer day and the beginning of the cycle in group 2. Conclusions: However, the fibrinogen, CRP and procalcitonin levels were significantly higher on the transfer day than at the beginning of the cycle. The results indicated that acute phase reactants or CA125 should not be used to determine the time of embryo transfer or predict pregnancy.
\end{abstract}

\section{Keywords}

Procalcitonin, CRP, Fibrinogen, CA125, Embryo Transfer, Pregnancy 


\section{Introduction}

Although a large number of embryos can be obtained from IVF patients, only $1 / 3$ of the patients become pregnant. This may be attributed to the transfer of embryos in an unsuitable period for implantation [1]. Synchronization between the embryo and endometrium is required for implantation. Therefore, to increase the chance of pregnancy, certain biomarkers could be useful for detecting endometrial receptivity and determining the best embryo transfer time. The use of such markers is important to reduce costs and avoid damaging the endometrium. Accordingly, C-reactive protein (CRP) and Cancer Antigen-125 (CA125) have been investigated as potential markers to determine endometrial receptivity in different studies. Various cytokines are secreted during implantation from the endometrium [2]. Leukemia inhibitory factor (LIF) and IL-1 are the most important biomarkers. LIF is involved in decidualization and implantation, and IL-1 allows the invasion of cytotrophoblasts by secreting Cyclooxygenase-2 (COX-2) [3]. Furthermore, the human leukocyte inhibitory factor (LIF) and Interleukin (IL-1) induce the secretion of direct acute phase reactants (APRs) [4]. Therefore, APRs released in response to inflammation in the body could be used for determining the implantation window [5]. The most well-known acute phase protein is CRP. In addition to CRP, there are various inflammatory substances secreted in the body. Hence, we evaluated changes not only in the levels of CRP but also fibrinogen (an acute phase protein) and procalcitonin (an inflammatory process marker during implantation). We also assessed another marker, CA125, which is secreted from the endometrium before implantation. CA125 is a glycoprotein that is secreted by the endometrium, and it enters the peripheral circulation [3] [4]. To determine the implantation period, some studies used the markers in fresh cycles to determine endometrial receptivity. In this study, we investigated the effect of these markers on frozen cycles to rule out the effects of induction and the oocyte pick-up (OPU) process, thus assessing only secretions induced by the endometrium. This study demonstrated the effect of APRs and CA125 levels in frozen cycles on pregnancy outcomes.

\section{Materials and Methods}

Between Feb and Sep 2018, 50 consecutive women who had their first frozen embryo transfer at a tertiary referral IVF center in Turkey were included in the study. All of the included patients provided informed consent, and were between 18 and 40 years of age. All of the patients have regular cycles. Patients who had tubal factor or anovulatory infertility are excluded from study. Patients who have autoimmune disease, systemic lupus erythamatosus, the patients with fever at and over $37.5^{\circ} \mathrm{C}$, the patients with elevated white blood cells, the patients with suspicion of infectious diseases; and the patients under antibiotherapy are excluded from study. Ethical approval was obtained from the University Ethics Committee. Informed consents were obtained from all women included in the study. Serum samples were collected from the patients for determining basal 
CRP, fibrinogen, procalcitonin, and CA125 levels on the second day of the cycle before the treatment started. On the transfer day, the serum samples of patients were re-collected, and CRP, fibrinogen, procalcitonin, and CA125 levels were re-evaluated. CRP, CA 125, fibrinogen and procalcitonin levels on the second day of the cycle are defined as CRP1, CA125 1, fibrinogen 1 and procalcitonin 1. CRP, CA 125, fibrinogen and procalcitonin levels on the transfer day are defined as CRP2, CA 125 2, fibrinogen 2 and procalcitonin 2. Endometrial preparation was initiated using estrogen (2 mg Estrofem; Novo Nordisk, Bagsvaerd, Denmark) on cycle day 2 - 3 following the transvaginal ultrasound. The endometrial preparation protocol began with $4 \mathrm{mg} /$ day of estrogen on days $1-4,6 \mathrm{mg} /$ day on days $5-8$, and $8 \mathrm{mg} /$ day from day 9 onwards. A second transvaginal ultrasound was performed following 10 days of estrogen treatment. Embryo transfer was scheduled in cases in which the endometrial thickness was at least $7 \mathrm{~mm}$. Progesterone was administered intramuscularly (50 mg Progestan; Koçak, Istanbul, Turkey) at a dose of $100 \mathrm{mg}$ for 5 complete days prior to embryo transfer. Progesterone was continued intramuscularly following the transfer $(50 \mathrm{mg}$ Progestan; Koçak, Istanbul, Turkey) for luteal support (up to the 12th week of pregnancy). The presence of pregnancy was assessed by BhCG (pregnancy test) test on the 14th day of the transfer. The presence of a gestational sac detected by ultrasound was the criteria for clinical pregnancy. In the study, the relationship between the pregnancy results of patients and fibrinogen, CRP, procalcitonin, and CA125 levels was evaluated. Primary outcome was clinical pregnancy rate.

The data were analyzed with IBM SPSS V23. The normal distribution of the data was determined by Shapiro-Wilk test. Mann-Whitney $U$ test and Wilcoxon test were used in the comparison of data that did not have a normal distribution. The results of the analysis were presented as the median (min-max). The significance level was fixed at $\mathrm{p}<0.05$.

\section{Results}

There were 16 patients pregnant (group 1) and 34 patients non-pregnant (group 2). 50 consecutive women were included in the study.

The median age of both groups was $32.26 \pm 4.65$ and $31.25 \pm 3.3$ and did not differ in terms of the presence of pregnancy $(\mathrm{p}=0.503)$ (Table 1$)$. The median estrogen, progesterone and LH level did not differ in terms of the presence of pregnancy $(\mathrm{p}=0.212, \mathrm{p}=0.664, \mathrm{p}=0.506)$. The median FSH level did not differ in terms of the pregnancy status $(\mathrm{p}=0.473)$. The median value was 7.3 for non-pregnant women and 8 for pregnant women. Endometrial thickness median values did not differ in terms of the pregnancy status $(\mathrm{p}=0.412)$. The median value was 9 for non-pregnant women and 9 for pregnant women. The number of retrieved oocyte median value did not differ according to the pregnancy status ( $\mathrm{p}$ $=0.472$ ). The median value was 12 for non-pregnant women and 10 for pregnant women. The number of antral follicles did not differ in terms of the pregnancy status $(p=0.338)$. The median value was 14 for non-pregnant women and 16 for 
Table 1. Comparison of parameters according to the pregnancy status.

\begin{tabular}{cccc}
\hline & Non-pregnant $(\mathrm{n}=34)$ & Pregnant $(\mathrm{n}=16)$ & $\mathrm{p}$ \\
\hline Age & $31(23-39) / 32.26 \pm 4.65$ & $31.5(26-37) / 31.25 \pm 3.3$ & 0.503 \\
Estrogen $^{*}$ & $199.5(5-359) / 199.88 \pm 87.45$ & $220.5(63-475) / 247.19 \pm 111.44$ & 0.212 \\
Progesterone* & $0.1(0.05-13) / 0.54 \pm 2.21$ & $0.15(0.05-1.09) / 0.23 \pm 0.26$ & 0.664 \\
LH* & $7.14(1.5-21) / 7.74 \pm 4.6$ & $8.45(1.6-26) / 9.59 \pm 6.86$ & 0.506 \\
Day 2 FSH level & $7.3(3.3-17.8) / 7.93 \pm 3.29$ & $8(4.7-14) / 8.41 \pm 2.85$ & 0.473 \\
Endometrial thickness & $9(5-14) / 9.18 \pm 1.86$ & $9(7-12) / 8.75 \pm 1.34$ & 0.412 \\
Number of Oocytes & $12(5-24) / 13.41 \pm 5.42$ & $10(4-31) / 13.25 \pm 7.81$ & 0.472 \\
Antral follicles Count & $14(6-20) / 13.74 \pm 4.82$ & $16(2-30) / 15.31 \pm 7.04$ & 0.338 \\
\hline
\end{tabular}

${ }^{*}$ Transfer day measurements.

pregnant women (Table 2).

CRP levels were different among the day 2 (CRP 1) and the transfer day (CRP2) ( $\mathrm{p}<0.001)$. For non-pregnant women the median CRP 1 level was 4.11, and the median CRP 2 level was 18.4. For pregnant women, the median CRP 1 level was 7.62, and the median CRP 2 level was 22.1. The median fibrinogen 1 and fibrinogen 2 levels were different among non-pregnant women $(\mathrm{p}<0.001)$. The median fibrinogen 1 level was 3.31, and the median fibrinogen 2 level was 4.5. The median fibrinogen 1 and fibrinogen 2 levels were also different among pregnant women $(\mathrm{p}=0.001)$. The median fibrinogen 1 level was 3.49 , and the median fibrinogen 2 level was 4.87 . The median procalcitonin 1 level was 0.03 , and the median procalcitonin 2 level was 0.04 with a significant difference ( $\mathrm{p}=$ 0.030) (Table 3). There was no statistically significant difference between the CA125 1 and CA125 2 levels of non-pregnant women or pregnant women ( $\mathrm{p}=$ $0.624, \mathrm{p}=0.173)$.

The median CRP 1 level did not differ according to the pregnancy status ( $\mathrm{p}=$ 0.595). The median value was 4.11 for non-pregnant women and 7.62 for pregnant women. The median CA125 1 level did not differ in terms of the pregnancy status $(\mathrm{p}=0.681)$. The median value was 13.35 for non-pregnant women and 11.39 for pregnant women. The median fibrinogen 1 level did not differ in terms of the pregnancy status $(\mathrm{p}=0.112)$. The median value was 3.31 for non-pregnant women and 3.49 for pregnant women. The median procalcitonin1level did not differ in terms of the pregnancy status $(p=0.708)$. The median value was 0.02 for non-pregnant women and 0.03 for pregnant women. The median CRP 2 level did not differ in terms of the pregnancy status $(\mathrm{p}=0.237)$. The median value was 18.4 for non-pregnant women and 22.1 for pregnant women. The median CA125 2 level did not differ in terms of the pregnancy status $(p=0.456)$. The median value was 13.18 for non-pregnant women and 12.86 for pregnant women. The median fibrinogen 2 level did not differ in terms of the pregnancy status $(\mathrm{p}=0.272)$. The median value was 4.5 for non-pregnant women and 4.87 for pregnant women. The median procalcitonin 2 level did not differ in terms of the 
Table 2. Comparison of the values according to pregnancy status.

\begin{tabular}{cccc}
\hline & Non-pregnant $(\mathrm{n}=34)$ & Pregnant $(\mathrm{n}=16)$ & $\mathrm{p}$ \\
\hline CRP 1 & $4.11(3.03-18.8) / 6.43 \pm 4.76$ & $7.62(3-47.7) / 11.32 \pm 12.6$ & 0.595 \\
CA125 1 & $13.35(4.96-43) / 15.22 \pm 9.21$ & $11.39(4.67-25) / 13.37 \pm 6.12$ & 0.681 \\
Fibrinogen 1 & $3.31(2.36-5.83) / 3.33 \pm 0.64$ & $3.49(2.89-4.02) / 3.54 \pm 0.38$ & 0.112 \\
Procalcitonin 1 & $0.02(0.02-0.08) / 0.03 \pm 0.01$ & $0.03(0-0.09) / 0.03 \pm 0.02$ & 0.708 \\
CRP 2 & $18.4(3.03-116) / 26.64 \pm 26.62$ & $22.1(7.1-113) / 33.93 \pm 29.65$ & 0.237 \\
CA125 2 & $13.18(4.8-38) / 15.33 \pm 8.34$ & $12.86(4.6-24.06) / 13.06 \pm 5.25$ & 0.456 \\
Fibrinogen 2 & $4.5(2.5-7.06) / 4.52 \pm 1.03$ & $4.87(3.62-6.29) / 4.8 \pm 0.77$ & 0.272 \\
Procalcitonin 2 & $0.03(0.02-0.08) / 0.03 \pm 0.02$ & $0.04(0.02-0.09) / 0.04 \pm 0.02$ & 0.398 \\
\hline
\end{tabular}

CRP 1, CA125 1, Fibrinogen 1 procalcitonin 1: Day 2 measurements; CRP 2, CA125 2, Fibrinogen 2, procalcitonin 2: Transfer day measurements.

Table 3. Comparison of the values at the beginning of cycle and transfer day.

\begin{tabular}{ccc}
\hline & Non-pregnant $(\mathrm{n}=34)$ & Pregnant $(\mathrm{n}=16)$ \\
\hline CRP 1 & $4.11(3.03-18.8) / 6.43 \pm 4.76$ & $7.62(3-47.7) / 11.32 \pm 12.6$ \\
CRP 2 & $18.4(3.03-116) / 26.64 \pm 26.62$ & $22.1(7.1-113) / 33.93 \pm 29.65$ \\
p & $<0,001$ & 0,005 \\
CA125 1 & $13.35(4.96-43) / 15.22 \pm 9.21$ & $11.39(4.67-25) / 13.37 \pm 6.12$ \\
CA125 2 & $13.18(4.8-38) / 15.33 \pm 8.34$ & $12.86(4.6-24.06) / 13.06 \pm 5.25$ \\
p & 0.624 & 0.173 \\
Fibrinogen 1 & $3.31(2.36-5.83) / 3.33 \pm 0.64$ & $3.49(2.89-4.02) / 3.54 \pm 0.38$ \\
Fibrinogen 2 & $4.5(2.5-7.06) / 4.52 \pm 1.03$ & $4.87(3.62-6.29) / 4.8 \pm 0.77$ \\
p & $<0.001$ & 0.001 \\
Procalcitonin1 & $0.02(0.02-0.08) / 0.03 \pm 0.01$ & $0.03(0-0.09) / 0.03 \pm 0.02$ \\
Procalcitonin 2 & $0.03(0.02-0.08) / 0.03 \pm 0.02$ & $0.04(0.02-0.09) / 0.04 \pm 0.02$ \\
p & 0.287 & 0.030 \\
\hline
\end{tabular}

pregnancy status $(\mathrm{p}=0.398)$. The median value was 0.03 for non-pregnant women and 0.04 for pregnant women.

\section{Discussion}

It is common to determine the implantation period of the endometrium with blood tests. This is because acquiring samples from the endometrium prepared for implantation could cause the endometrium to deteriorate, and it is costly compared with blood tests.CA125 level in the blood may be examined to determine endometrial receptivity. It is produced from the endometrium and transferred directly to the serum. Noci et al. [5] found the CA125 levels of pregnant patients on OPU day were lower than those of non-pregnant patients. Brenden- 
berger et al. [6] reported that there was no relationship between CA125 levels and pregnancy results. Tavmergen et al. demonstrated that CA125 levels were significantly higher in pregnant women, consistent with the two previous studies [7] [8] [9]. Tavmergen et al. [9] reported that CA125 at a dose of $10 \mathrm{IU} / \mathrm{mL}$ and above on OPU day was important for the prediction of pregnancy. In the present study, there was no relationship between CA125 levels and pregnancy results. As the implantation process is an inflammatory process, we investigated whether there was an effect on pregnancy by identifying APRs in the serum. CRP is a pentameric protein secreted by the liver in response to inflammation [10]. CRP is increased during induction, and the increase in CRP can be further enhanced when hCG is administered [11]. In addition, after the OPU process, CRP is increased [2]. More importantly, the implantation of the embryo into the endometrium is an inflammatory process. The embryo is prepared for endometrial implantation by secreting IL-1 [12]. CRP is increased in the blood in response to IL-1 [10]. Almagor et al. reported that there was no difference between the CRP levels of pregnant and non-pregnant patients on OPU day and on the day of transfer; however, the transfer/retrievable oocyte rate was significantly lower in pregnant patients than in non-pregnant patients [2]. Levin et al. revealed that CRP elevation at each stage could adversely affect IVF results. However, this relationship has not been observed in other studies [11] [13]. Seckin et al. examined high-sensitivity CRP (hs-CRP) levels at the beginning of induction and on the 7th day after transfer in fresh cycles and found no significant difference between pregnant and non-pregnant patients [14]. Fibrinogen is also an APR. Devranoğlu et al. reported lower levels of fibrinogen in pregnant patients with factor V Leiden mutation on the hCG day [15]. However, we did not observe any difference in fibrinogen levels between pregnant and non-pregnant patients. Procalcitonin is a marker that has been used in the follow-up of various inflammatory processes in recent years. Procalcitonin is a peptide made up of 116 amino acids [16]. It is encoded by the CALC-1 geneon chromosome 11. In addition to infections, procalcitonin is expressed in neuroendocrine tissues. Procalcitonin released from thyroid $\mathrm{C}$ cells is converted to calcitonin with some modifications. However, in inflammatory processes, procalcitonin is produced by the expression of the CALC-1 gene in other tissues [17]. Although its functions remain unclear, procalcitonin is a promising marker in inflammatory processes [18]. Considering that implantation is an inflammatory process, we investigated whether procalcitonin should be used as a marker to determine the success of implantation. We found that procalcitonin, CRP, and fibrinogen were significantly increased on the day of transfer. However, the reason for this increase remains unclear. The results suggest that this increase was not related to the occurrence of pregnancy.

There are some limitations in our study. First of all the following period is short so we could not investigate the effect of APRs in abortus rates. And the study population may be larger. 


\section{Conclusion}

In conclusion, the results indicated that APRs and CA125 should not be used to determine the time of embryo transfer or pregnancy status. Nevertheless, prospective studies involving larger patient populations are needed to confirm the results.

\section{Compliance with Ethical Standards}

Ethical approval was obtained from Ondokuz Mayıs University.

\section{Conflicts of Interest}

All authors declared no conflict of interest.

\section{References}

[1] Paulson, R.J., Sauer, M.V. and Lobo, R.A. (1990) Factors Affecting Embryo Implantation after Human in Vitro Fertilization: A Hypothesis. American Journal of $O b$ stetrics \& Gynecology, 163, 2020-2023. https://doi.org/10.1016/0002-9378(90)90790-E

[2] Zeimet, A.G., Offner, F.A., Müller-Holzner, E., et al. (1998) Peritoneum and Tissues of the Female Reproductive Tract as Physiological Sources of CA-125. Tumor Biology, 19, 275-282. https://doi.org/10.1159/000030018

[3] Bischof, P., Tseng, L., Brioschi, P.A. and Herrmann, W.L. (1986) Cancer Antigen 125 Is Produced by Human Endometrial Stromal Cells. Human Reproduction, 1, 423-426. https://doi.org/10.1093/oxfordjournals.humrep.a136445

[4] Noci, I., Maggi, M., Biagiotti, R., D’Agata, A., Criscuoli, L., et al. (1999) Serum CA-125 Values on the Day of Oocyte Retrieval Are Not Predictive of Subsequent Pregnancy with In- Vitro Fertilization. Human Reproduction, 14, 1773-1776. https://doi.org/10.1093/humrep/14.7.1773

[5] Almagor, M., Hazav, A. and Yaffe, H. (2018) The Levels of C-Reactive Protein in Women Treated by IVF. Human Reproduction, 19, 104-106. https://doi.org/10.1093/humrep/deh036

[6] Brandenberger, A.W., Bersinger, N.A., Huber, P.R., Berger, E., Glanzmann, P. and Birkhaeuser, M.H. (1998) CA-125 Concentrations in the Serum and Pregnancy Outcome in IVF Cycles. Journal of Assisted Reproduction and Genetics, 15, 390-394. https://doi.org/10.1023/A:1022589101648

[7] Chryssikopoulos, A., Mantzavinos, T., Kanakas, N., Karagouni, E., Dotsika, E., et al. (1996) Correlation of Serum and Follicular Fluid Concentrations of Placental Protein 14 and CA-125 in in Vitro Fertilization-Embryo Transfer Patients. Fertility and Sterility, 66, 599-603. https://doi.org/10.1016/S0015-0282(16)58574-4

[8] Kathleen, A., Miller, B.S., Jeffrey, L., Deaton, M.D., Donald, E., Pittaway, M.D., et al. (1996) Evaluation of Serum CA 125 Concentrations as Predictors of Pregnancy with Human in Vitro Fertilization. Fertility and Sterility, 65, 1184-1189. https://doi.org/10.1016/S0015-0282(16)58336-8

[9] Tavmergen, E., Sendag, F., Nazan, E., et al. (2001) Value of Serum CA-125 Concentrations as Predictors of Pregnancy in Assisted Reproduction Cycles. Human Reproduction, 16, 1129-1134. https://doi.org/10.1093/humrep/16.6.1129

[10] Pepys, M.B. and Hirschfield, G.M. (2003) C-Reactive Protein: A Critical Update. Journal of Clinical Investigation, 111, 1805-1812. 
https://doi.org/10.1172/JCI200318921

[11] Orvieto, R., Chen, R., Ashkenazi, J., Ben-Haroush, A., Bar, J., et al. (2004) C-Reactive Protein Levels in Patients Undergoing Controlled Ovarian Hyperstimulation for IVF Cycle. Human Reproduction, 19, 357-359. https://doi.org/10.1093/humrep/deh089

[12] Lindhard, A., Bentin-Ley, U., Ravn, V., Islin, H., Hviid, T., Rex, S., et al. (2002) Biochemical Evaluation of Endometrial Function at the Time of Implantation. Fertility and Sterility, 78, 221-233. https://doi.org/10.1016/S0015-0282(02)03240-5

[13] Nardo, L.G., et al. (2008) Low Grade Inflammation, as Evidenced by Basal High Sensitivity CRP, Is Not Correlated to Outcome Measures in IVF. Journal of Assisted Reproduction and Genetics, 25, 383-388. https://doi.org/10.1007/s10815-008-9253-y

[14] Seckin, B., Ozaksit, G., Batioglu, S., Murat Ozel, M.A. and Senturk, B. (2012) The Relationship between the Change in Serum High Sensitivity C-Reactive Protein Levels and IVF Success. Gynecological Endocrinology, 28, 418-421. https://doi.org/10.3109/09513590.2011.633653

[15] Devranoglu, B., et al. (2018) Coagulation Tests at Trigger Day in Patients with Factor V Leiden Mutation to Predict Implantation Failure. Gynecology Obstetrics \& Reproductive Medicine, 24, 76-81. https://doi.org/10.21613/GORM.2017.754

[16] Ming, J., Adil, I. and Khan, P. (2010) Procalcitonin: Uses in the Clinical Laboratory for the Diagnosis of Sepsis. Lab Medicine, 41, 173-177. https://doi.org/10.1309/LMQ2GRR4QLFKHCH9

[17] Linscheid, P., Seboek, D., Nylen, E.S., et al. (2003) In Vitro and in Vivo Calcitonin I Gene Expression in Parenchymal Cells: A Novel Product of Human Adipose Tissue. Endocrinology, 144, 5578-5584. https://doi.org/10.1210/en.2003-0854

[18] Becker, K.L., Snider, R., et al. (2008) Procalcitonin Assay in Systemic Inflammation, Infection, and Sepsis: Clinical Utility and Limitations. Critical Care Medicine, 36, 941-952. https://doi.org/10.1097/CCM.0B013E318165BABB 